\title{
On fully three-dimensional resistive wall mode and feedback stabilization computations
}

\author{
E. Strumberger, P. Merkel, M. Sempf, and S. Günter \\ Max-Planck-Institut für Plasmaphysik, 85748 Garching, Germany \\ EURATOM Association
}

\begin{abstract}
Resistive walls, located closely to the plasma boundary, reduce the growth rates of external kink modes to resistive time scales. For such slowly growing resistive wall modes the stabilization by an active feedback system becomes feasible. The fully three-dimensional stability code STARWALL, and the feedback optimization code OPTIM have been developed [P. Merkel, et al., 21st IAEA Fusion Energy Conference 2006, Chengdu, China, paper TH/P3-8] to compute the growth rates of resistive wall modes in the presence of non-axisymmetric, multiply-connected wall structures and to model the active feedback stabilization of these modes. In order to demonstrate the capabilities of the codes and to study the effect of the toroidal mode coupling caused by multiply-connected wall structures, the codes are applied to test equilibria using the resistive wall structures currently under debate for ITER [M. Shimada, et al., Nuclear Fusion 47, S1 (2007)] and ASDEX Upgrade [W. Köppendörfer, et al., Proc. of the 16th Symp. on Fusion Technology, London, 1990, Vol. 1, p. 208].
\end{abstract}




\section{INTRODUCTION}

A high plasma beta is a necessary condition for a working power plant. However, if neoclassical tearing modes are avoided by a suitable choice of the current profile, external kink modes can limit the achievable plasma beta in tokamak devices. These are ideal instabilities, growing on a very short time scale of $t \sim 10^{-6} \mathrm{~s}$. They could be stabilized by an ideal wall sufficiently close to the plasma boundary. But, in presence of a resistive wall the instabilities grow on a resistive time scale of $t \sim 10^{-2}-10^{-3}$ s. Since these resistive wall modes (RWMs) grow on a longer time scale, their feedback stabilization is technically feasible.

While tokamak plasmas are approximately axisymmetric, a realistic external wall will have a complex three-dimensional shape. Designs for walls in ITER [1,2] and for an additional, closely fitting wall in ASDEX Upgrade (AUG) [3, 4] show multiply-connected structures (i.e., with holes), as shown in Fig. 1. These walls and the magnetic fields generated by the feedback coils cause a violation of the axisymmetry resulting in a toroidal mode coupling, and, therefore, making a three-dimensional numerical treatment of the feedback stabilization problem necessary.

Already existing codes for RWMs and feedback studies are either completely two-dimensional, such as the MARS-F code [5] and the KINX code [6], or they use a three-dimensional model for the wall and the feedback coils only, such as the MARS-F/CARIDDI code [7] and the VALEN code [8]. All these codes neglect the toroidal mode coupling in their plasma stability calculations.

For self-consistent, three-dimensional feedback stabilization studies, the stability code STARWALL and the feedback optimization code OPTIM have been developed [9]. Taking several toroidal harmonics simultanously into account, the STARWALL code is able to compute openloop and with a given set of feedback parameters also closed-loop resistive wall eigenmodes in the presence of non-axisymmetric, multiply-connected wall structures. In order to find an optimal set of feedback parameters making the system stable an optimization procedure (OPTIM 
code) has been developed.

The goal of this paper is twofold. First of all, the capabilities of the codes are demonstrated by studies of 3D effects caused by multiply-connected wall structures. Especially, for the first time the toroidal mode coupling is taken into account. Secondly, the strength of the toroidal mode coupling is compared for the ITER and AUG wall structures, which are currently under debate. In Sec. II a brief introduction into the solution method of the three-dimensional feedback stabilization problem is presented (for details see Refs 9,10). Sec. III contains a test case study. Two similar test equilibria have been chosen in order to study the 3D effects for ITER and AUG wall structures. In Sec. III A the test equilibria are described, whereas in Sec. III B their ideal stability properties are investigated. Stability studies with resistive wall structures are presented in Sec. III C, while the feedback stabilization of the RWMs is discussed in Sec. III D. Finally, in Sec. IV the results are summerized and an outlook for further investigations is given.

\section{THREE-DIMENSIONAL STABILITY STUDIES}

The energy principle of the ideal MHD can be extended to the description of RWMs. In this case the kinetic energy $W_{\mathrm{k}}$, the potential energy of the plasma $W_{\mathrm{p}}$, and the energy flux across the plasma surface $W_{\mathrm{s}}$, have to satisfy

$$
-\gamma^{2} W_{\mathrm{k}}(\vec{\xi}, \vec{\xi})-W_{\mathrm{p}}(\vec{\xi}, \vec{\xi})-W_{\mathrm{s}}=0
$$

This equation holds for eigenvalues $\gamma$, and normal eigenmodes $\vec{\xi}$ assuming a time dependence $\mathrm{e}^{\gamma t}$. The general form of the energy flux

$$
W_{\mathrm{s}}=\frac{1}{2 \mu_{0}} \int_{\mathrm{S}_{\mathrm{p}}} d f(\vec{n} \cdot \vec{\xi})\left(\vec{B} \cdot \vec{B}_{0}\right)
$$

can be expressed by

$$
W_{\mathrm{s}}=\frac{1}{2 \mu_{0}} \int_{\mathrm{V}_{\mathrm{vac}}} d V B^{2}+\frac{1}{2 \gamma \sigma d} \int_{\mathrm{S}_{\mathrm{w}}} d f J_{w}^{2}
$$


with permeability $\mu_{0}$, plasma surface $\mathrm{S}_{\mathrm{p}}$, volume of the vacuum region $V_{\text {vac }}$, exterior normal $\vec{n}$, displacement vector $\vec{\xi}$, equilibrium magnetic field $\vec{B}_{0}$, vacuum magnetic field perturbation $\vec{B}=\nabla \times \vec{A}$, and the resistive wall quantities: surface $\mathrm{S}_{\mathrm{w}}$, surface current $\vec{J}_{\mathrm{w}}$, conductivity $\sigma$, and wall thickness $d$.

Using flux co-ordinates $(s, u, v)$, the displacement vector $\vec{\xi}=\left(\xi^{s}, \xi^{u}, \xi^{v}\right)$ is expanded in $(u, v)$ Fourier space:

$$
\vec{\xi}(\vec{r}, t)=\mathrm{e}^{\gamma t} \sum_{\mathrm{m}, \mathrm{n}} \vec{\xi}_{\mathrm{m}, \mathrm{n}}^{s}(s) \sin [2 \pi(\mathrm{m} u+\mathrm{n} v)]+\vec{\xi}_{\mathrm{m}, \mathrm{n}}^{c}(s) \cos [2 \pi(\mathrm{m} u+\mathrm{n} v)] .
$$

The radial co-ordinate $s$ labels the magnetic surfaces (normalized toroidal flux), $(u, v)$ are poloidal and toroidal magnetic co-ordinates on the surfaces, and $(m, n)$ are the poloidal and toroidal Fourier indices. In radial direction a finite element approach is used.

In the vacuum region the vector potential $\vec{A}$ has to satisfy Maxwell's equations and boundary conditions at the plasma-vacuum interface and the conducting wall. For the resistive wall the boundary condition follows from Faraday's and Ohm's laws. With the time dependence $\vec{A} \sim \mathrm{e}^{\gamma t}$, one finds for the thin wall approximation

$$
\vec{n} \times \vec{A}= \begin{cases}-(\vec{n} \cdot \vec{\xi}) \vec{B}_{0} & \text { on } S_{\mathrm{p}} \text { (plasma-vacuum interface) } \\ 0 & \text { on } S_{\mathrm{w}} \text { (ideal wall) } \\ \text { or } & \\ -\frac{1}{\sigma d \gamma} \vec{n} \times \vec{J}_{\mathrm{w}} & \text { on } S_{\mathrm{w}}(\text { resistive wall) }\end{cases}
$$

With the CAS3D stability code [11] internal and external eigenmodes of 3D equilibria can be calculated without and with an ideally conducting wall $(\sigma=\infty)$. With the STARWALL code resistive wall modes are computed. Assuming the RWM is growing sufficiently slow, the kinetic energy of the plasma perturbation is neglected: $W_{\mathrm{k}}(\vec{\xi}, \vec{\xi})=0$. The plasma potential energy matrix $W_{\mathrm{p}}(\vec{\xi}, \vec{\xi})$ is provided by the CAS3D code. The contribution $W_{\mathrm{s}}$ of the vacuum region containing the resistive wall is determined by the magnetic field perturbation $\vec{B}=\nabla \times \vec{A}$. The vector potential $\vec{A}$ can be generated by surface current densities $\vec{j}_{\mathrm{s}}$ on the plasma-vacuum interface, on the resistive wall, and in the feedback coils. A divergence-free surface current density can be derived from a current potential $\Phi: \vec{j}_{\mathrm{s}}=\vec{n} \times \nabla \Phi$. Discretizing the boundaries 
into triangles and assuming a constant current density on the triangles, the current potential $\Phi$ is determined by a finite element method using a variational procedure [9].

\section{APPLICATIONS}

\section{A. EQUILIBRIA}

The numerical procedures are applied to two similar test equilibria which are unstable with respect to $n=1$ and $n=2$ external kink modes. Fixed-boundary equilibria are calculated with the VMEC code [12] for chosen plasma boundaries, pressure and advanced safety factor profiles shown in Fig. 2 and Fig. 3. The shapes of these profiles and the form of the plasma boundaries are chosen intentionally identical in order to compare the effect of the toroidal mode coupling, caused by different multiply-connected wall structures. Whereas, the following parameters have been chosen according to the ITER and AUG design: ITER/AUG: major radius $R_{0}=6.40 / 1.71 \mathrm{~m}$, minor radius $a_{0}=1.17 / 0.44 \mathrm{~m}$, plasma current $I_{\mathrm{p}}=9.2 / 0.8 \mathrm{MA}$, magnetic field strength $B_{0}=5.24 / 1.97 \mathrm{~T}$, plasma pressure at the magnetic axis $p_{\mathrm{a}}=530 / 73 \mathrm{kPa}$, volume-averaged plasma pressure $\langle p\rangle=297 / 41 \mathrm{kPa}$, volume-averaged plasma beta $\langle\beta\rangle=2.53 / 2.50 \%$, and beta normalized $\beta_{\mathrm{N}}=2.51 / 2.62$.

\section{B. IDEAL STABILITY}

Without a conducting wall the equilibria are unstable with respect to external kink modes. Because of the axisymmetry of the equilibria, the eigenmodes are classified by one toroidal index $n$ only. With the CAS3D stability code $n=1$ and $n=2$ kink modes are calculated. As an example, Fig. 4 shows the leading harmonics $\xi_{\mathrm{m}, \mathrm{n}}^{\mathrm{s}, \mathrm{s}}, \xi_{\mathrm{m}, \mathrm{n}}^{\mathrm{s}, \mathrm{c}}$ of the radial displacment $\xi^{\mathrm{s}}$ versus the toroidal flux s for the $n=1$ (Fig. 4a) and n=2 (Fig. 4b) kink modes of the ITER case. The growth rates of these modes are $\gamma=190001 / \mathrm{s}$ for $\mathrm{n}=1$ and $\gamma=72011 / \mathrm{s}$ for $\mathrm{n}=2$.

In order to stabilize such modes, conducting walls have been designed for ITER, and for AUG 
additional wall structures are under consideration. In Figs 1 and 2 preliminary designs of the walls and feedback coils are shown.

The ITER wall is poloidally and toroidally closed. There are 45 holes at the low field side in approximately equidistant poloidal and toroidal distances. The outer wall of the ITER wall structure has been neglected because its additional stabilizing effect is only of the order of $5-10 \%$.

The additional conducting wall for AUG consists of a toroidally closed segment, located at the low field side, where the amplitudes of the kink modes are largest. The relatively large area of the 16 holes in the AUG wall reduces the stabilizing effect of the wall considerably. Therefore, it is advantageous that the relative plasma-wall distance of the AUG wall $\Delta_{\mathrm{w}} / a_{0}$ will be much smaller compared to the ITER case (ITER/AUG: $\Delta_{\mathrm{w}} / a_{0}=0.50 / 0.17$, see Fig. 2).

\section{STABILITY STUDIES WITH RESISTIVE WALL STRUCTURES}

Analogous to the method presented in Ref. 13, the resistive wall mode and feedback stabilization problem is separated into two parts: the open- and the closed-loop problem. In the open-loop part, a complete set of eigenfunctions of the plasma-resistive wall system is determined. The feedback coils are included in the resistive wall configuration. They are additional passive resistive elements without an applied external voltage.

Taking the finite resistivity of the wall structures into account, the growth rates of the resistive wall modes for the ITER and AUG examples are calculated with the STARWALL code for two different wall structures (axisymmetric, closed walls and multiply-connected walls). For ITER, the closed wall is obtained from the multiply-connected wall by closing the holes, while for AUG the wall segment is also extended in poloidal direction. It is important to note at this place that for the $3 \mathrm{D}$ case both torodial Fourier indices $n=1$ and $n=2$ are simultaniously taken into account. For ITER as well as for AUG four unstable resistive wall modes have been found. Table I summarizes the normalized growth rates $\gamma \mu_{0} \sigma d$ of these modes. Comparing the results 
for the multiply-connected walls (column: 3D) and for axisymmetric, closed walls (column: 2D) the eigenvalues for the most unstable modes are found to be $4.4 \mathrm{~m}^{-1}$ for the $3 \mathrm{D}$ ITER case and $10.32 \mathrm{~m}^{-1}$ for the $3 \mathrm{D}$ AUG case. The eigenvalues of the $2 \mathrm{D}$ closed wall configurations are two-fold degenerate, while they are non-degenerate in the 3D case. As expected, the AUG wall breaks the axisymmetry stronger than the ITER wall. Note that the toroidal Fourier index $n$ given in Table I characterizes the modes in case of an axisymmetric, closed wall. In the 3D case, the modes are dominated by the given n, but also the other toroidal Fourier index contributes to these modes.

Figure 5 shows the leading harmonics $\xi_{\mathrm{m}, \mathrm{n}}^{\mathrm{s}, \mathrm{s}}, \xi_{\mathrm{m}, \mathrm{n}}^{\mathrm{s}, \mathrm{c}}$ of the eigenfunctions for the above-given eigenvalues which represent the most unstable modes. Shown is the radial component $\xi$ s of the displacement vector $\vec{\xi}$ as a function of the radial co-ordinate $s$ for ITER (Fig. 5a) and AUG (Fig. 5b). While for ITER the leading harmonics have the same toroidal Fourier index $(n=2)$, for AUG Fourier harmonics with $n=1$ and $n=2$ contribute to the eigenfunction. That is, the complex 3D structure of the AUG wall leads to a toroidal mode coupling.

Finally, Figs $6 a, b$ show contour plots of the current potential $\Phi$ on the wall for the most unstable modes of ITER and AUG. The vertical and horizontal axes of the flat projection of the wall indicate the poloidal and toroidal directions, respectively. The contour lines are the current lines of the surface current density. The figures illustrate the reduction of the stabilizing wall currents by the holes, leading to an increase of the growth rates (see Table I).

\section{FEEDBACK STABILIZATION}

In the closed-loop part, which includes sensor loops and feedback logics, voltages are applied to the feedback coils. In the voltage-control feedback model, which is used in this study, all coils and sensors are grouped into one or more toroidal bands. Each coil band $\mathrm{k}(\mathrm{k}=1, \ldots, \mathrm{K})$ is linked to each sensor band $1(\mathrm{l}=1, \ldots, \mathrm{L})$ via a proportional gain matrix $G^{\mathrm{k}, \mathrm{l}}$. These matrices map the sensor signals onto voltages which are applied to the coils. In addition, a cascade control 
is applied to each coil, which feeds back the current flowing in a coil onto the coil voltage by an additional gain factor $-R_{\mathrm{k}}^{\mathrm{C}}$ which is equal for each member of the $\mathrm{k}$-th coil band. This is equivalent to changing the coil's resistance artificially by $R_{\mathrm{k}}^{\mathrm{C}}$. The voltage applied to the $\mathrm{i}$-th coil in the k-th toroidal band is then given by

$$
U_{\mathrm{i}}^{\mathrm{k}}=\sum_{\mathrm{l}=1}^{\mathrm{L}} \sum_{\mathrm{j}=1}^{\mathrm{N}_{1}^{\mathrm{S}}} G_{\mathrm{i}, \mathrm{j}}^{\mathrm{k}, 1} S_{\mathrm{j}}^{\mathrm{l}}-R_{\mathrm{k}}^{\mathrm{C}} I_{\mathrm{i}}^{\mathrm{k}}, \quad \mathrm{i}=1, \ldots, \mathrm{N}_{\mathrm{k}}^{\mathrm{C}}
$$

where $G_{\mathrm{i}, \mathrm{j}}^{\mathrm{k}, 1}$ denotes the element $(\mathrm{i}, \mathrm{j})$ of the matrix $G^{\mathrm{k}, 1} . \mathrm{N}_{\mathrm{k}}^{\mathrm{C}}$ and $\mathrm{N}_{1}^{\mathrm{S}}$ are the total numbers of coils in the k-th toroidal band and of the sensors in the 1-th sensor band, respectively. $I_{\mathrm{i}}^{\mathrm{k}}$ is the current flowing in the i-th coil, and $S_{\mathrm{j}}^{1}$ the signal measured by the $\mathrm{j}$-th sensor in that band.

The problem is to find $G^{\mathrm{k}, 1}$ and $R_{\mathrm{k}}^{\mathrm{C}}$ so that the system is stable. However, to run the closed-loop computations repeatedly for different $G^{\mathrm{k}, 1}$ and $R_{\mathrm{k}}^{\mathrm{C}}$ until all eigenvalues have negative real parts is computationally very tedious. Instead, the problem is reduced in a physically reasonable manner. First of all, the gain matrices $G^{\mathrm{k}, \mathrm{l}}$ are constructed in such a way that each coil band produces a field classified by one toroidal Fourier index $\mathrm{n}$ in response to measured perturbations with the same $\mathrm{n}$. This response is a linear combination of a response having the same toroidal phase as the perturbation, and another one which is phase shifted by $90 / \mathrm{n}$ degrees. Thus,

$$
G_{\mathrm{i}, \mathrm{j}}^{\mathrm{k}, \mathrm{l}}=\sum_{\mathrm{n}} \alpha_{\mathrm{n}}^{\mathrm{k}, 1} \cos \left(\mathrm{n} \varphi_{\mathrm{i}, \mathrm{j}}^{\mathrm{k}, \mathrm{l}}\right)+\beta_{\mathrm{n}}^{\mathrm{k}, \mathrm{l}} \sin \left(\mathrm{n} \varphi_{\mathrm{i}, \mathrm{j}}^{\mathrm{k}, \mathrm{l}}\right),
$$

where $\varphi_{\mathrm{i}, \mathrm{j}}^{\mathrm{k}, \mathrm{l}}$ is the toroidal angle between coil i of coil band $\mathrm{k}$ and sensor $\mathrm{j}$ of sensor band 1 . The sum runs over all n's to be controlled. Here, $n=1,2$ is used. Secondly, the dimension of the system is reduced by projecting the system's matrix onto a subspace. This subspace is defined by those open-loop eigenvectors which are considered to be most important for the problem. In order to determine the remaining free parameters $\alpha_{\mathrm{n}}^{\mathrm{k}, 1}, \beta_{\mathrm{n}}^{\mathrm{k}, 1}$, and $R_{\mathrm{k}}^{\mathrm{C}}$ of the reduced problem the OPTIM code is applied. It uses the so-called gradient bundle method [14] for the nonlinear search of the feedback parameters with the objective to minimize the real parts of all eigenvalues [9]. Having self-consistently solved the problem for the reduced system does not necessarily 
imply that all modes are stable for the full system. For a final check the full system is solved with the STARWALL code using the parameters obtained for the reduced system.

Figure 7 shows the complex normalized eigenvalues $\gamma \mu_{0} \sigma d$ for the STARWALL open-loop, and STARWALL/OPTIM closed-loop computations obtained for ITER (Fig. 7a) and AUG (Fig. 7b). For the STARWALL open- and closed-loop computations, 5199 (ITER) and 9022 (AUG) eigenvectors have been used, whereas the dimension of the reduced OPTIM subspace is 1024 (ITER) and 1536 (AUG). The figures show that, in both cases, a stabilizing parameter set has been found, as all eigenvalues' real parts are negative. As expected, there are moderate deviations between the eigenvalues obtained with reduced and full-size calculations.

\section{SUMMARY AND OUTLOOK}

In this paper a brief report on the current status and the first results of fully $3 \mathrm{D}$ resistive wall mode and feedback stabilization computations are presented. Two similar test equilibria have been used to investigate the toroidal mode coupling caused by multiply-connected wall structures which are currently under debate for ITER and AUG. Taking torodial mode coupling into account, four unstable resistive wall modes have been found for both test cases. Feedback stabilization was applicable for all the cases. The results also indicate that for ITER toroidal mode coupling may be insignificant. However, in comparison to the respective closed wall, the holes in the wall lead to a considerable increase of the growth rate for both, ITER and AUG. Furthermore, the computations show that the complex structure of the additional AUG wall segment leads to a significant coupling of the $n=1$ and $n=2$ Fourier harmonics.

Clearly, a detailed analysis of the parameter space of the equilibria, dimensional effects (2D vs. 3D), and the sensitivity with respect to the feedback parameters is needed. However, this is beyond the scope of this paper but currenly under intensive investigation. 


\section{ACKNOWLEDGEMENTS}

The authors would like to thank C. Nührenberg for providing the CAS3D code, T. Bertoncelli, J. Bialek and J. Menard for providing the wall and coil data for AUG and ITER, respectively, and W. Suttrop for useful discussions. 


\section{References}

[1] M. Shimada, D. Campbell, V. Mukhovatav, et al., Nulear Fusion 47, S1 (2007).

[2] T.C. Hender, J.C. Wesley, J. Bialek, et al., Nulear Fusion 47, S128 (2007).

[3] W. Köppendörfer, M. Blaumoser, H. Bruhns, et al., Proceedings of the 16th Symposium on Fusion Technology, London, 1990, edited by B.E. Keen, M. Huguet, R. Hemsworth (Elsevier, Amsterdam, 1991), Vol. 1, p. 208.

[4] W. Suttrop, T. Bertoncelli, V. Bobkov, et al., Proceedings of the 34th EPS Conf. on Plasma Phys., Warsaw, 2007, edited by P. Gasior, J. Wolowski, [Europhys. Conf. Abstr. 31F, P$5.119(2007)]$.

[5] Y. Liu, A. Bondeson, Y. Gribov, A. Polevoi, Nuclear Fusion 44, 232 (2004).

[6] L. Degtyarev, A. Martynov, S. Medvedev, F. Troyon, L. Villard, R. Gruber, Comp. Phys. Commun. 103, 10 (1997).

[7] F. Villone, G. Rubinacci, Y.Q. Liu, Y. Gribov, Proceedings of the 32nd EPS Conf. on Plasma Phys. and Contr. Fusion combined with the 8th Int. Workshop on Fast Ignition of Fusion Targets, Tarragona, edited by C. Hidalgo, B. Ph. van Milligen, [Europhys. Conf. Abstr. 29C, P-5.049 (2005)].

[8] J. Bialek, A. Boozer, M.E. Mauel, A. Navratil, Phys. Plasmas 8, 2170 (2001).

[9] P. Merkel, M. Sempf, 21st IAEA Fusion Energy Conference 2006, Chengdu, China, paper TH/P3-8 (International Atomic Energy Agency, Vienna, 2006).

[10] P. Merkel, C. Nührenberg, E. Strumberger, Proceedings of the 31st EPS Conf. on Plasma Phys., London, 2004, edited by P. Norreys, H. Hutchinson [Europhys. Conf. Abstr. 28G, P-1.208 (2004)]. 
[11] C. Nührenberg, Phys. Plasmas 3, 2401 (1996).

[12] S.P. Hirshman, W.I. van Rij, P. Merkel, Comput. Phys. Commun. 43, 143 (1986).

[13] M.S. Chu, M.S. Chance, A.H. Glasser, M. Okabayashi, Nuclear Fusion 43, 441 (2003).

[14] J.V. Burke, A.S. Lewis, M.L. Overton, Lin. Algebra Appl. 351-352, 117 (2002). 


\section{FIGURE CAPTIONS}

Fig. 1: 3D representation of preliminarily designed resistive wall structures and feedback coils for (a) ITER and (b) AUG.

Fig. 2: Cross-sections of (a) ITER and (b) AUG test equilibria showing flux surfaces and plasma boundaries, as well as the locations of resistive walls, feedback coils (ITER: 2 toroidal bands, AUG: 3 toroidal bands), and sensors (ITER/AUG: 1 toroidal band) measuring the poloidal magnetic field perturbation.

Fig. 3: (a) Pressure and (b) $q$-profiles as function of $\rho_{\text {tor }}=\sqrt{s}$ ( $s=$ normalized toroidal flux) for ITER (solid line) and AUG (dashed line) test equilibria.

Fig. 4: Harmonics $\xi_{m, n}^{\mathrm{s,s}}, \xi_{m, n}^{\mathrm{s}, \mathrm{c}}$ of the radial displacement $\xi^{s}$ as function of $s$ for the unstable (a) $n=1(\gamma=190001 / s)$ and (b) $n=2(\gamma=72011 / s)$ kink modes of the ITER test equilibrium without external walls and coils.

Fig. 5: Harmonics $\xi_{m, n}^{\mathrm{s, \textrm {s }}}, \xi_{m, n}^{\mathrm{s}, \mathrm{c}}$ of the radial displacement $\xi^{s}$ as function of $s$ for the most unstable RWM of the (a) ITER $\left(\gamma \mu_{0} \sigma d=4.41 / \mathrm{m}\right)$ and the (b) AUG test equillib$\operatorname{rium}\left(\gamma \mu_{0} \sigma d=10.321 / \mathrm{m}\right)$.

Fig. 6: (Color online) Contour plots of the current potential $\Phi$ on the (a) ITER and (b) AUG wall. The current potential distributions are produced by the RWMs shown in Figs $5 \mathrm{a}$ and $5 \mathrm{~b}$. The vertical and horizontal axes of the flat projections of the walls indicate the poloidal and toroidal directions, respectively.

Fig. 7: Open and closed-loop eigenvalue spectra for (a) ITER and (b) AUG. 
ITER

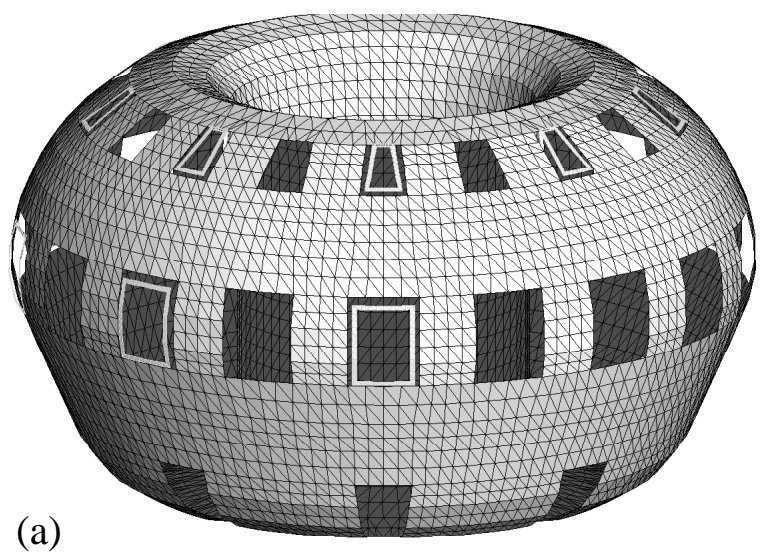

ASDEX Upgrade

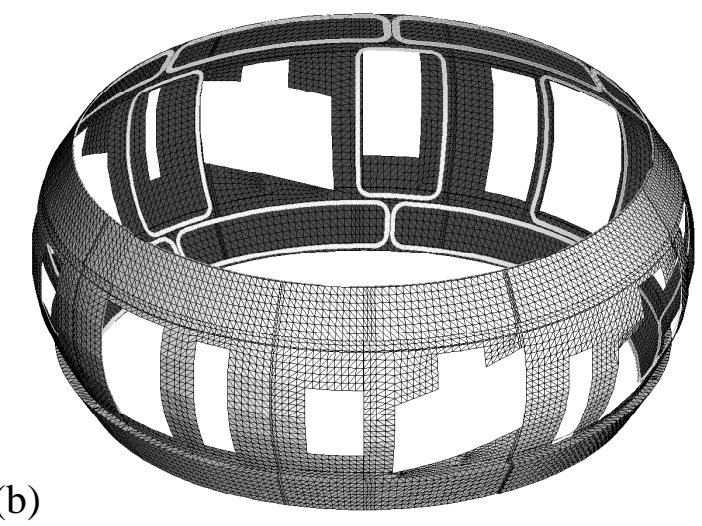

FIG. 1 

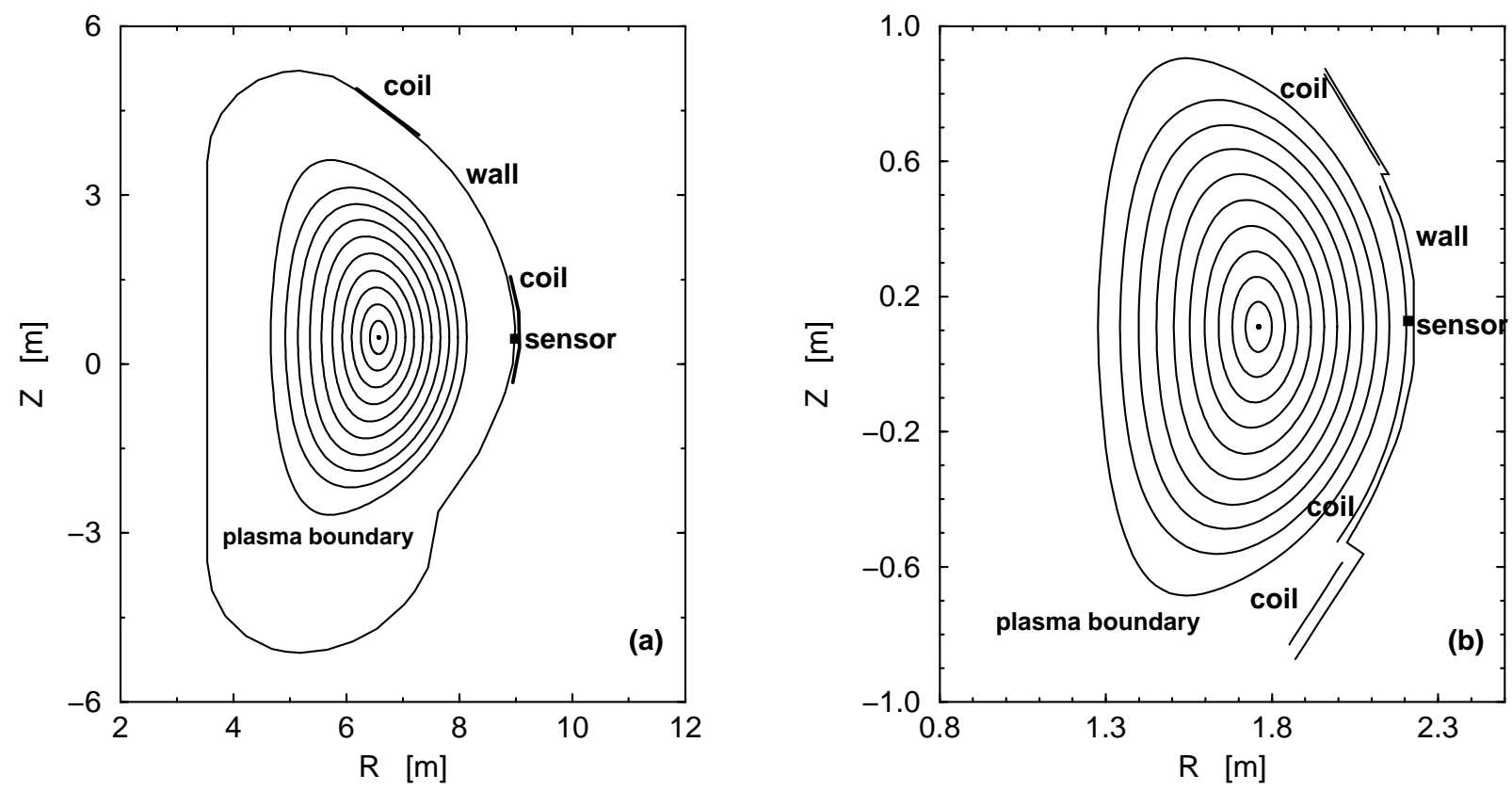

FIG. 2 

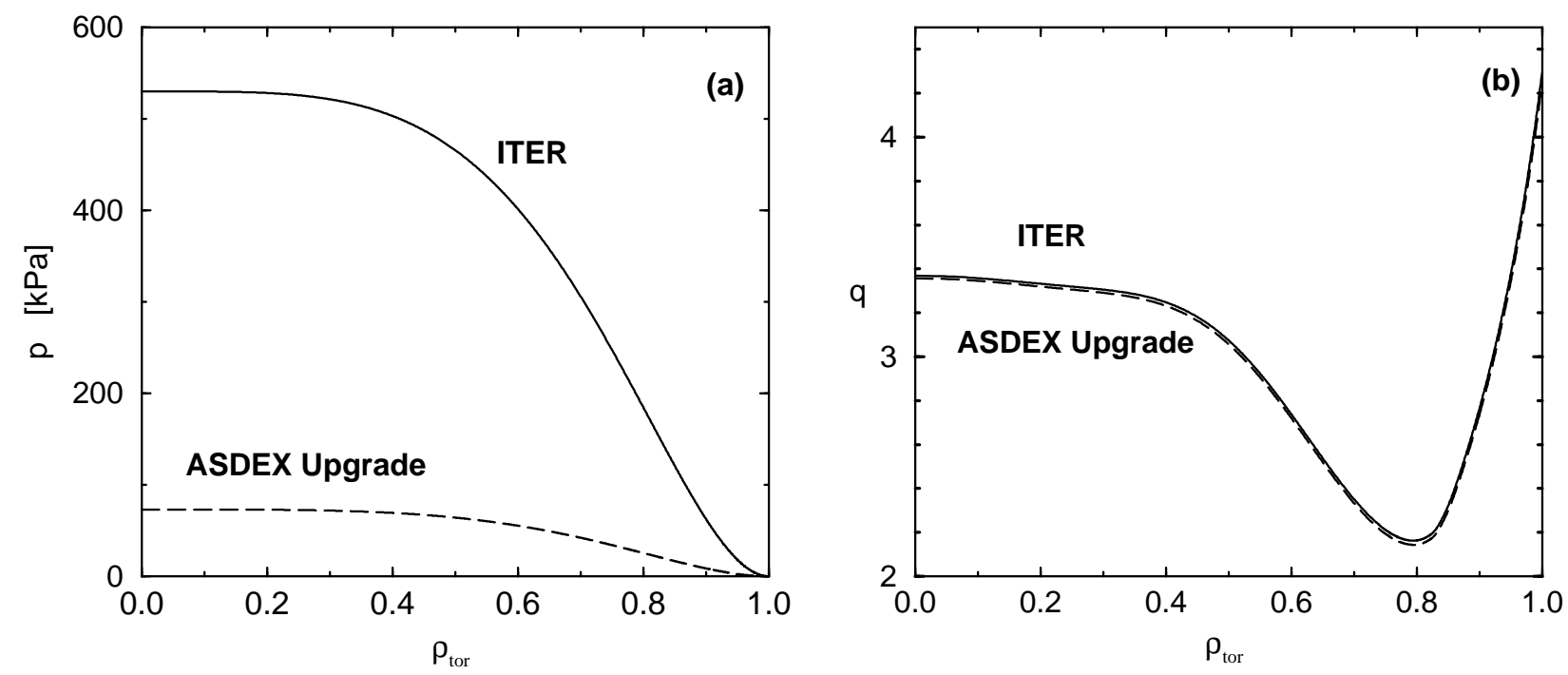

FIG. 3 

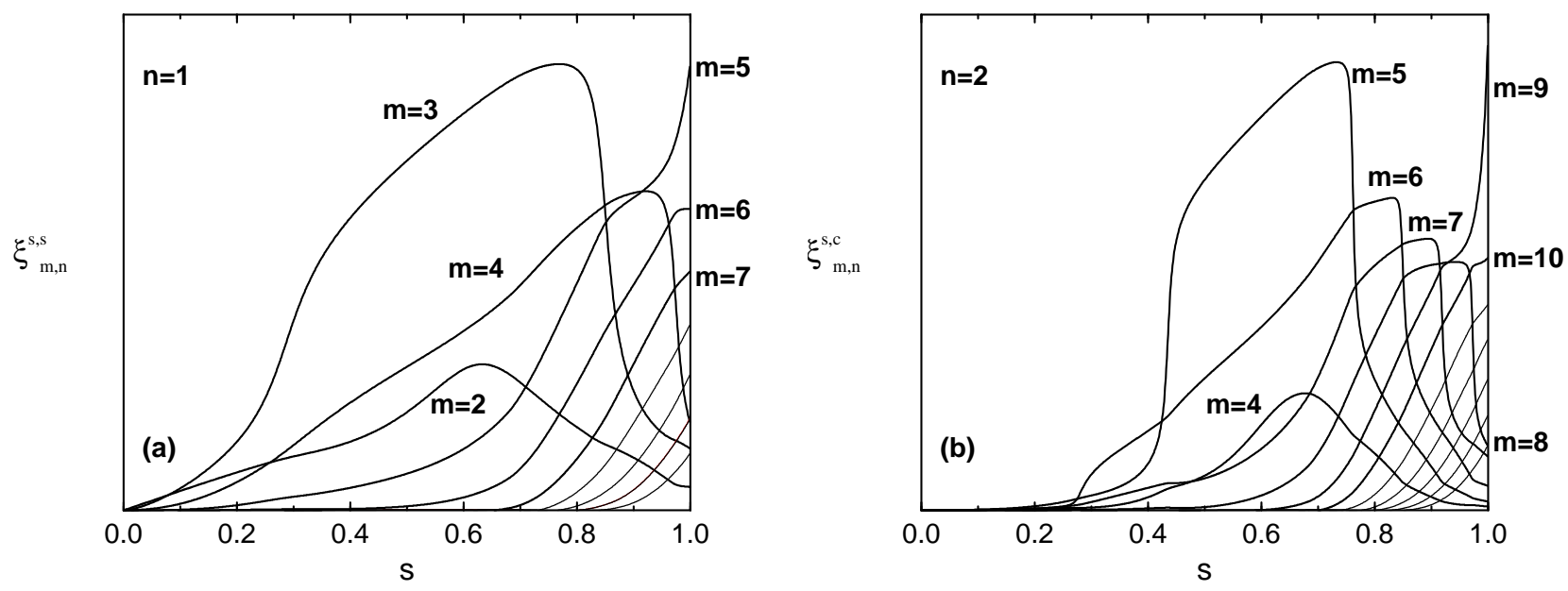

FIG. 4 

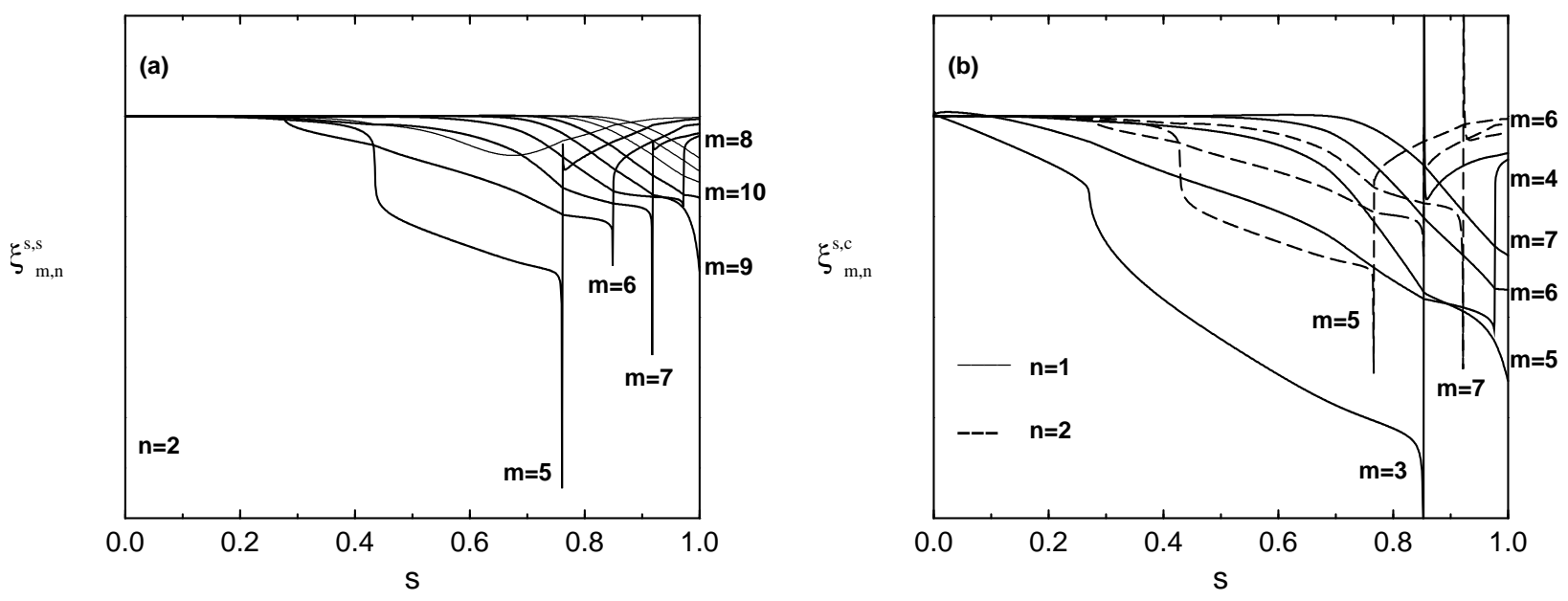

FIG. 5 


\section{ITER}

(a)

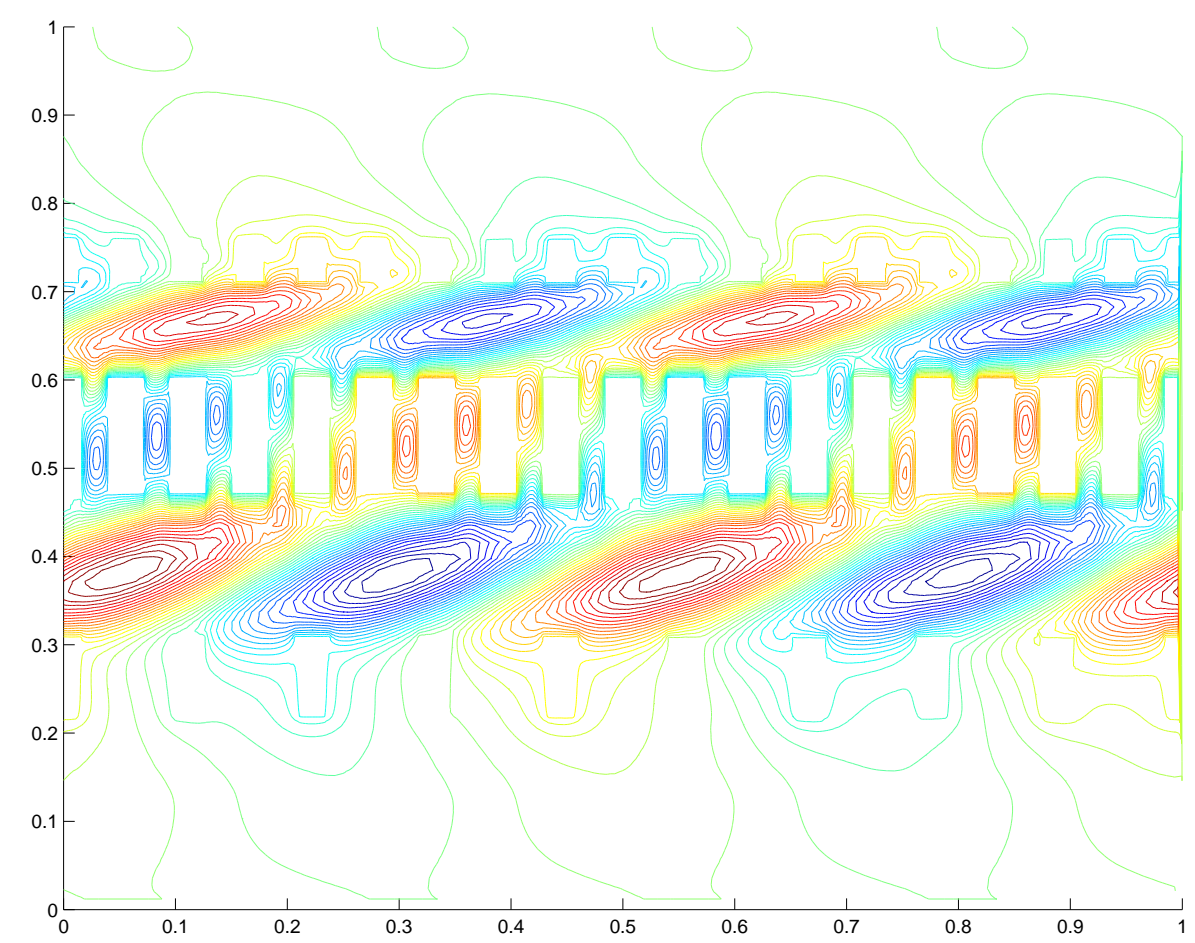

ASDEX Upgrade

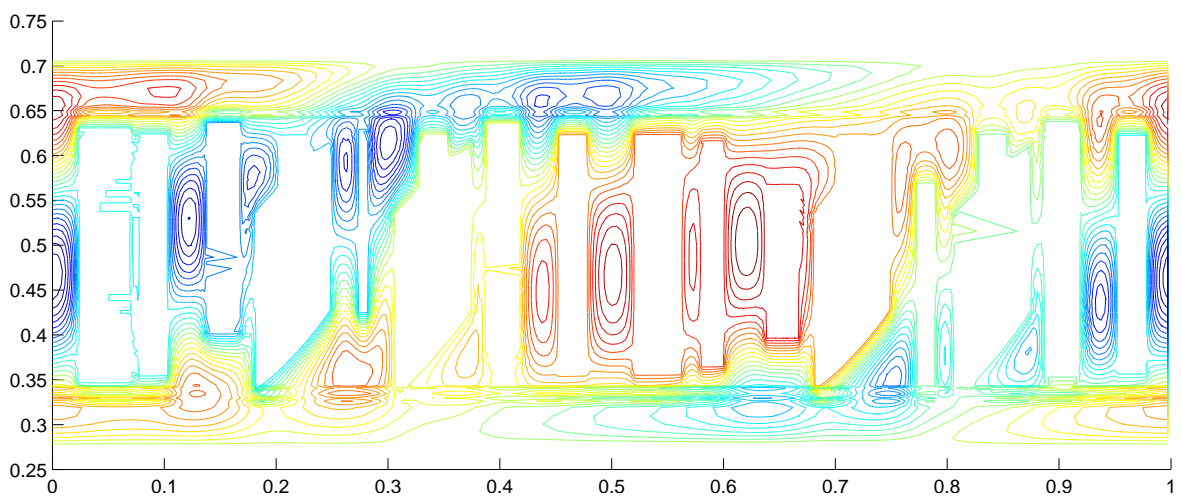

FIG. 6 

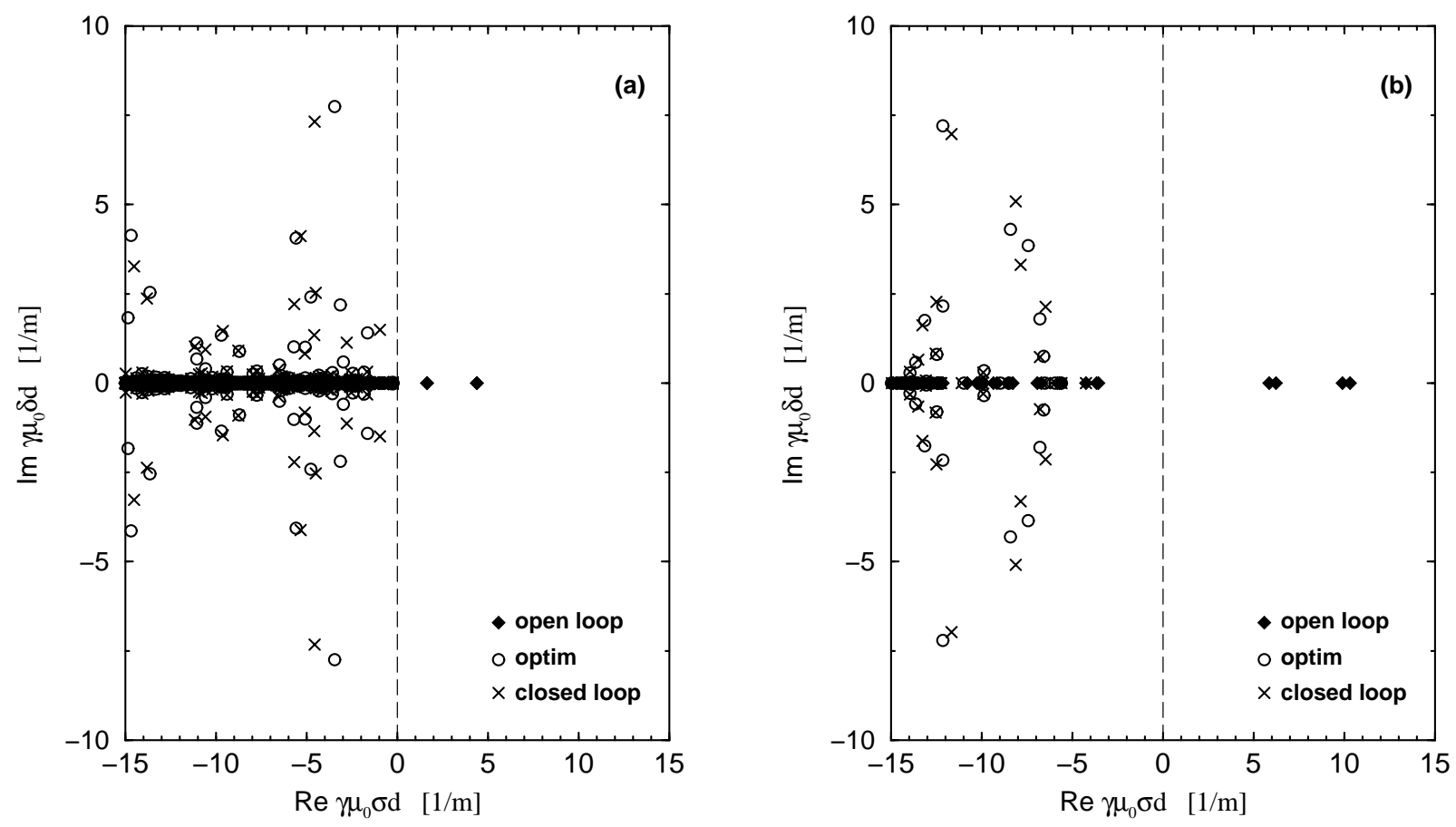

FIG. 7 
Table I: unstable normalized

\begin{tabular}{rrrrr}
\multicolumn{5}{c}{ eigenvalues $\gamma \mu_{0} \sigma d[1 / \mathrm{m}]$} \\
\hline \hline & ITER & ITER & AUG & AUG \\
$\mathrm{n}$ & 2D & 3D & 2D & 3D \\
\hline 1 & 0.79 & 1.65 & 1.79 & 10.32 \\
1 & 0.79 & 1.64 & 1.79 & 9.91 \\
2 & 1.65 & 4.40 & 1.69 & 6.24 \\
2 & 1.65 & 4.38 & 1.69 & 5.86 \\
\hline \hline
\end{tabular}

